\title{
Über die Verwendung von Maple für die Simulation von Mechanismen
}

\author{
ENGIN CAN
}

\begin{abstract}
Maple is used to do numerical computation, plot graphs and do exact symbolic manipulations and word processing. This paper demonstrates how Maple can be used for the simulation of mechanisms. This offers the possibility for students to become familiar with this particular section of mathematical modelling. The mechanism under consideration is a so-called F-mechanisms, i.e., a planar parallel 3-RRR robot with three synchronously driven cranks. It turns out that at this example it is not possible to find the poses of the moving triangle exactly by graphical methods with traditional instruments only. Hence, numerical methods are essential for the analysis of motions which can be performed by an F-mechanism.
\end{abstract}

Key words and phrases: Maple, scientific computing, mathematical modelling, planar mechanism, F-mechanism, planar parallel 3-RRR-robot.

ZDM Subject Classification: N60, P50, R20.

\section{Einführung}

F-Mechanismen wurden erstmals in [1] und [2] vorgestellt und analysiert. Es sind dies schnelllaufende ebene Mechanismen mit modifizierbaren Zwangläufen, die auf parallelen Robotern mit simultan angetriebenen Kurbeln basieren. Auch in [5] wird die Kinematik derartiger Roboter behandelt; die Website [3] bietet eine

Diese Arbeit wurde mit der Projektnummer 2014-17-02-001 durch den Forschungsfonds der Sakarya Universität unterstützt.

Copyright (C) 2015 by University of Debrecen 
interaktiv steuerbare Simulation paralleler ebener Manipulatoren. Im folgenden wiederholen wir die charakteristischen Merkmale von F-Mechanismen:

\subsection{Definition}

Unter einem Fehrer-Mechanismus (kurz: F-Mechanismus) verstehen wir im folgenden eine 8-gliedrige kinematische Kette mit folgenden Merkmalen (siehe Abb.1):

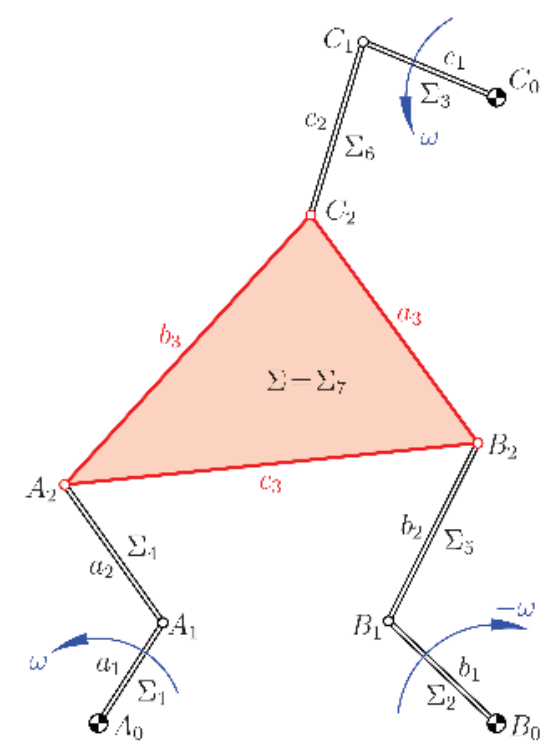

Abbildung 1. Schema eines (gegensinnigen) F-Mechanismus

(1) Es gibt drei mit der gleichen Winkelgeschwindigkeit $\omega$ umlaufende Antriebskurbeln $\Sigma_{1}, \Sigma_{2}, \Sigma_{3}$ mit den Lagerpunkte $A_{0}, B_{0}$ bzw. $C_{0}$ und den Kurbelendpunkten $A_{1}, B_{1}$ bzw. $C_{1}$. Deren Längen seien mit $a_{1}, b_{1}$ bzw. $c_{1}$ bezeichnet. Die Kurbeln $\Sigma_{1}$ und $\Sigma_{3}$ drehen stets in demselben (mathematisch positiven) Sinn.

Die Kurbel $\Sigma_{2}$ kann positiv oder negativ drehen. Bei negativem Drehsinn dieser Kurbel sprechen wir von einem gegensinnigen F-Mechanismus, sonst von einem gleichsinnigen. 


$$
\text { "02-can" — 2015/5/21 — 23:29 — page } 23 \text { — \#3 }
$$

(2) Das Gangsystem $\Sigma=\Sigma_{7}$ enthält drei Punkte $A_{2}, B_{2}, C_{2}$, die mit den Kurbelenden $A_{1}, B_{1}$ bzw. $C_{1}$ jeweils durch Stäbe $\Sigma_{4}, \Sigma_{5}$ bzw. $\Sigma_{6}$ der Längen $a_{2}, b_{2}$ bzw. $c_{2}$ verbunden sind.

(3) Durch zwei kontinuierlich verstellbare Phasenverschiebungen $\delta_{b}$ und $\delta_{c}$ zwischen den Kurbeln $\Sigma_{2}$ und $\Sigma_{1}$ bzw. zwischen $\Sigma_{3}$ und $\Sigma_{1}$ sind während des Betriebes Modifikationen des Zwanglaufes $\Sigma_{7} / \Sigma_{0}$ möglich.

Dabei definieren wir $\delta_{c}$ als von der Zeit $t$ unabhängige Differenz der Drehwinkel der Kurbeln $C_{0} C_{1} \subset \Sigma_{3}$ und $A_{0} A_{1} \subset \Sigma_{1}$, also $\delta_{c}=\varphi_{30}-\varphi_{10}$, und im gleichsinnigen Fall analog $\delta_{b}=\varphi_{20}-\varphi_{10}$. Im gegensinnigen Fall messen wir die Phasenverschiebung $\delta_{b}$ als jenen Winkel, den in der Ausgangslage $t:=\varphi_{10}=0$ die von $A_{0}$ nach rechts weisende Kurbel $A_{0} A_{1}$ mit der Kurbel $B_{0} B_{1}$ einschließt, also $\delta_{b}=\varphi_{20}(0)-\varphi_{10}(0)$.

Wir bezeichnen gelegentlich die Seitenlängen des Rastdreiecks mit $a_{0}, b_{0}$ bzw. $c_{0}$ und jene des Gangdreiecks mit $a_{3}, b_{3}$ bzw. $c_{3}$.

\section{BEMERKUNG.}

(1) Wir sprechen im folgenden vom Rast- oder Lagerdreieck $A_{0} B_{0} C_{0}$ und vom Gangdreieck $A_{2} B_{2} C_{2}$, obwohl diese Punktetripel auch kollinear sein dürfen.

(2) Die Forderung nach gleicher Antriebsgeschwindigkeit für alle Kurbeln lässt sich natürlich auch mechanisch realisieren durch geeignete Riemen- oder Zahnrädergetriebe oder im gleichsinnigen Fall auch durch Parallelkurbeln.

\subsection{Formenreichtum der Zwangläufen}

Die folgenden zwei Beispiele sollen die Vielfalt der durch einen F-Mechanismus erzeugbaren Zwangläufe illustrieren:

BEISPIEL 1. Abbildung 2 zeigt einen vollständig umlaufenden F-Mechanismus zu den Abmessungen

$$
\begin{array}{ccc}
\overline{A_{0} B_{0}}=300, & \overline{B_{0} C_{0}}=371.2, & \overline{A_{0} C_{0}}=404.0, \\
a_{1}=84\left(177^{\circ}\right), & b_{1}=104\left(103^{\circ}\right), & c_{1}=107\left(312^{\circ}\right), \\
a_{2}=185, & b_{2}=117, & c_{2}=185, \\
\overline{A_{2} B_{2}}=205, & \overline{B_{2} C_{2}}=250, & \overline{A_{2} C_{2}}=117 .
\end{array}
$$

Dieses Getriebe wurde mit Hilfe der Getriebeentwurf-Software SAM 6.1 der Firma ARTAS-Engineering Software analysiert. Das der Bildschirmausgabe entnommene Bild zeigt als Resultat die Bahnkurve des Punktes 7 der Gangebene. 


$$
\text { "02-can" — 2015/5/21 — 23:29 — page } 24 \text { — \#4 }
$$

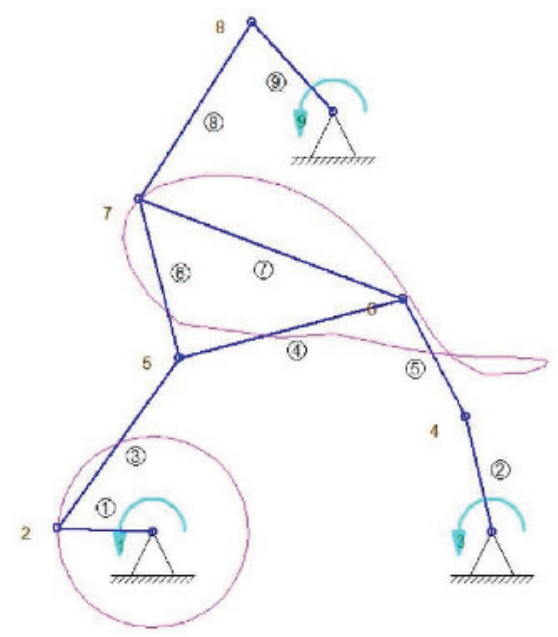

Abbildung 2. Beispiel eines gleichsinnigen F-Mechanismus(Ausgabe der Getriebeentwurf-Software SAM 6.1)

Beispiel 2. Allgemeiner Zwanglauf:

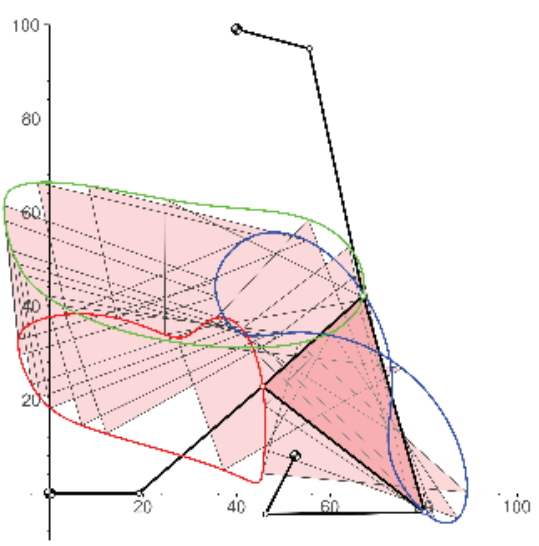

Abbildung 3. Zwanglauf des gegensinnigen F-Mechanismus aus dem Beispiel 2

Die Abbildung 3 zeigt den Zwanglauf eines gegensinnigen F-Mechanismus, welcher in der Folge mehrfach als Beispiel benutzt wird, z.B. in den Abbildungen 5, 7 und 8. Die zugehörigen Abmessungen lauten: 
Rastdreieck: $A_{0}=(0.0,0.0), B_{0}=(52.5,8.0), C_{0}=(40.0,99.0)$, Kurbellängen: $a_{1}=19.0, b_{1}=14.0, c_{1}=16.0$,

Phasenverschiebungen: $\delta_{b}=243^{\circ}, \delta_{c}=-15^{\circ}$,

Armlängen: $a_{2}=35.0, b_{2}=34.0, c_{2}=54.0$,

Gangdreieck: $A_{2}=(0.0,0.0), B_{2}=(40.0,18.0), C_{2}=(-7.0,28.0)$.

\section{Darstellung des Zwanglaufs $\Sigma_{7} / \Sigma_{0}$}

Nun wenden wir uns dem Zwanglauf $\Sigma_{7} / \Sigma_{0}$ bei einem F-Mechanismus (siehe Abb.4) zu und diskutieren, wie wir dessen Positionen berechnen können.Die Bewegung eines beliebigen Punktes $X \in \Sigma_{7}$ gegenüber $\Sigma_{0}$ ist ansetzbar als

$$
\mathbf{x}_{0}(t)=\mathbf{u}_{0}(t)+A(t) \mathbf{x}_{7} \operatorname{mit} A(t)=\left(\begin{array}{cc}
\cos \varphi_{70} & -\sin \varphi_{70} \\
\sin \varphi_{70} & \cos \varphi_{70}
\end{array}\right)
$$

Darin ist $\mathbf{u}_{0}(t)$ die im Rastkoordinatensystem $\left(x_{0}, y_{0}\right)$ dargestellte und zunächst unbekannte Bahn des Ursprungs $U$ des Gangkoordinatensystems $\left(x_{7}, y_{7}\right)$ in $\Sigma_{7}$. Mit $\varphi 70(t)$ ist der Drehwinkel des Gangkoordinatensystems gegenüber dem Rastkoordinatensystem in $\Sigma_{0}$ bezeichnet. $\mathbf{x}_{7}$ sind die Gangkoordinaten eines Punktes $X \in \Sigma_{7}$ und $\mathbf{x}_{0}$ die Rastkoordinaten des augenblicklich deckungsgleichen Punktes aus $\Sigma_{0}$.

Für eine numerische Darstellung von $\Sigma_{7} / \Sigma_{0}$ setzen wir in der orthogonalen Matrix $A(t)$ die trigonometrischen Funktionen $\sin \varphi_{70}$ und $\cos \varphi_{70}$ in bekannter Weise als rationale Funktionen an mittels

$$
f:=\tan \frac{\varphi_{70}(t)}{2}
$$

und daher

$$
\sin \varphi_{70}=\frac{2 f}{1+f^{2}}, \cos \varphi_{70}=\frac{1-f^{2}}{1+f^{2}}
$$

also

$$
A(t)=\frac{1}{1+f^{2}}\left(\begin{array}{cc}
1-f^{2} & -2 f \\
2 f & 1-f^{2}
\end{array}\right)
$$


mit

$$
f=f(t) .
$$

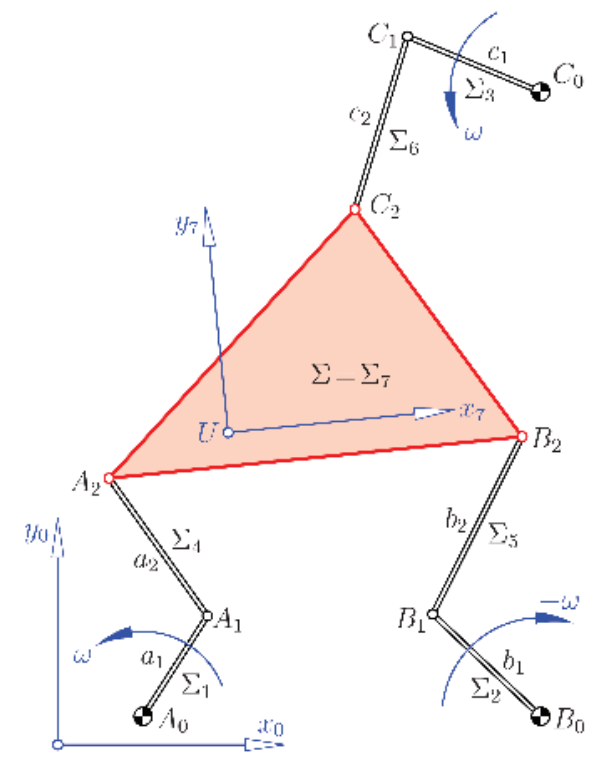

Abbildung 4. Schema eines (gegensinnigen) F-mechanismus

Wir bezeichnen die konstanten Gangkoordinaten der Punkte $A_{2}, B_{2}$ bzw. $C_{2}$ in $\Sigma_{7}$ mit $\mathbf{a}_{2 \mid 7}, \mathbf{b}_{2 \mid 7}$ und $\mathbf{c}_{2 \mid 7}$. Damit können wir ihre Bahnen im Rastsystem darstellen als

$$
\begin{aligned}
\mathbf{a}_{2 \mid 0}(t) & =\mathbf{u}_{0}(t)+A(t) \mathbf{a}_{2 \mid 7}, \\
\mathbf{b}_{2 \mid 0}(t) & =\mathbf{u}_{0}(t)+A(t) \mathbf{b}_{2 \mid 7}, \\
\mathbf{c}_{2 \mid 0}(t) & =\mathbf{u}_{0}(t)+A(t) \mathbf{c}_{2 \mid 7} .
\end{aligned}
$$

Wegen der gegebenen Winkelgeschwindigkeiten der drei Antriebskurbeln sind die Bahnen der Kurbelenden $A_{1}, B_{1}$ bzw. $C_{1}$ ansetzbar als

$$
\mathbf{a}_{1 \mid 0}(t)=a_{1}\left(\begin{array}{c}
\cos \omega t \\
\sin \omega t
\end{array}\right)
$$




$$
\begin{gathered}
\mathbf{b}_{1 \mid 0}(t)=b_{1}\left(\begin{array}{c}
\cos \left(\mp \omega t+\delta_{b}\right) \\
\sin \left(\mp \omega t+\delta_{b}\right)
\end{array}\right) \\
\mathbf{c}_{1 \mid 0}(t)=c_{1}\left(\begin{array}{c}
\cos \left(\omega t+\delta_{c}\right) \\
\sin \left(\omega t+\delta_{c}\right)
\end{array}\right)
\end{gathered}
$$

mit $a_{1}, b_{1}, c_{1}$ als Kurbellängen (Abb. 4).

Die Bewegung $\Sigma_{7} / \Sigma_{0}$ wird nun festgelegt durch die konstanten Längen $a_{2}, b_{2}$ bzw. $c_{2}$ der Arme $A_{1} A_{2}, B_{1} B_{2}$ bzw. $C_{1} C_{2}$. Dies ergibt die quadratischen Bedingungen

$$
\begin{aligned}
& \left(a_{2 \mid 0}(t)-a_{1 \mid 0}(t)\right)^{2}=a_{2}^{2}=\text { konst. } \\
& \left(b_{2 \mid 0}(t)-b_{1 \mid 0}(t)\right)^{2}=b_{2}^{2}=\text { konst. } \\
& \left(c_{2 \mid 0}(t)-c_{1 \mid 0}(t)\right)^{2}=c_{2}^{2}=\text { konst. }
\end{aligned}
$$

Aus diesen drei Gleichungen müssen die Unbekannten $\left(f, u_{x}, u_{y}\right)$ mit

$$
f=\tan \frac{\varphi_{70}(t)}{2} \text { und } \mathbf{u}_{0}=\left(\begin{array}{l}
u_{x} \\
u_{y}
\end{array}\right)
$$

berechnet werden.

Der geometrische Hintergrund dieses Eliminationsprozesses ist wie folgt: Bei vorübergehend festgehaltenem Antriebsparameter $t$ liegt $C_{2}$ einerseits auf einem Kreis mit der Mitte $C_{1}$, andererseits auf der Koppelkurve mit den Lagerpunkten $A_{1}$ und $B_{1}$ und der Koppel $A_{2} B_{2}$. Im generischen Fall sind Koppelkurven trizirkulare Kurven 6. Ordnung (Siehe 6, S.106). Nach dem Satz von Bèzout gibt es abgesehen von den je dreifach zu zählenden absoluten Kreispunkten $6 \cdot 2-2 \cdot 3=6$ mögliche Punkte $C_{2}$, wobei natürlich auch paarweise konjugiert Schnittpunkte auftreten können.

\section{Eine Maple-Prozedur zur Berechnung des Zwanglaufs $\Sigma_{7} / \Sigma_{0}$}

$\mathrm{Zu}$ Beginn werden die notwendigen Daten eingegeben, also die Koordinaten des Rastdreiecks und des Gangdreiecks sowie die Längen der Kurbeln und Arme, dann die Phasenverschiebungen samt Angabe, ob der F-Mechanismus gleichsinnig oder gegensinnig ist. Dazu kommen noch die Grenzen für den Antriebswinkel sowie die Anzahl der zu berechnenden Zwischenlagen. Aus programmiertechnischen Gründen sind einige Abweichungen von der früheren Bezeichnung unvermeidlich. 
So wird z.B. der Tangens des halben Drehwinkels $\varphi_{70}$ der Gangebene mit $t$ oder $t_{\text {neu }}$ bezeichnet, während die Antriebswinkel der einzelnen Kurbeln ff1, ff2 bzw. ff3 heißen.

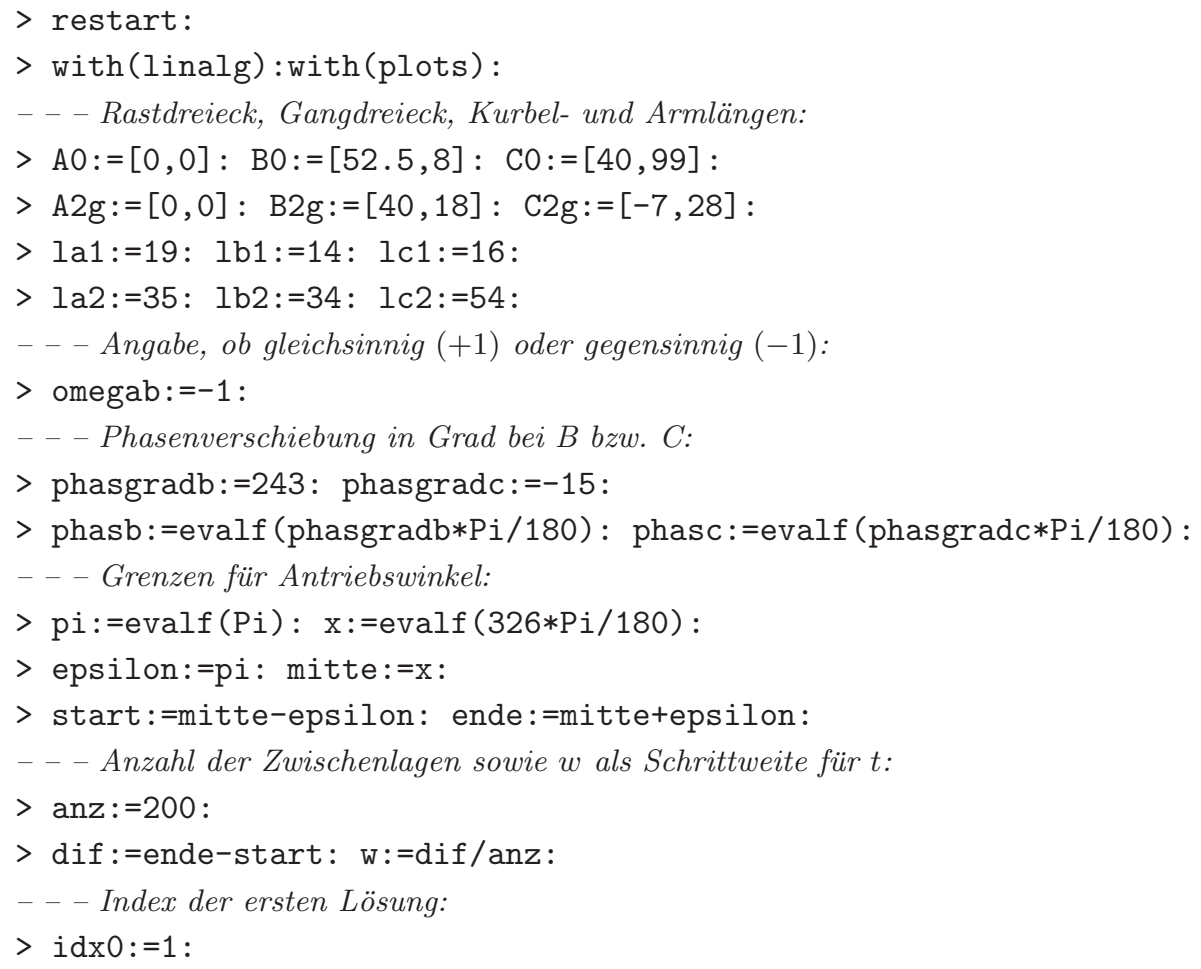

Nun setzen wir die Punkte $A_{1}, B_{1}$ und $C_{1}$ in Abhängigkeit vom Tangens $f_{1}$, $f_{2}$ bzw. $f_{3}$ der halben Antriebswinkel an. Daneben wird der Drehwinkel $t:=\varphi_{70}$ eingeführt. Ferner führen wir den Schiebvektor trans $=(u, v):=\mathbf{u}_{0}$ ein sowie die orthogonale Matrix dreh $:=A$ und setzen in Abhängigkeit davon die Ecken des Gangdreiecks an:

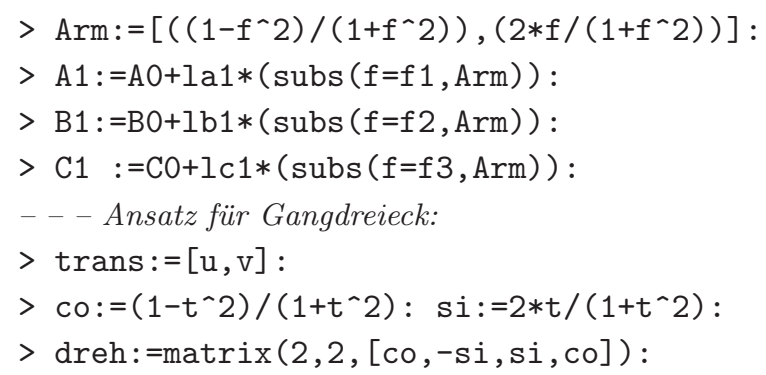




$$
\text { "02-can" — 2015/5/21 — 23:29 — page } 29 \text { — \#9 }
$$

$>$ A2:=simplify (matadd (evalm(dreh \&* A2g), trans)):

$>$ B2:=simplify (matadd (evalm(dreh \&* B2g), trans)):

$>\mathrm{C} 2:=\operatorname{simplify}($ matadd (evalm(dreh \&* C2g), trans)):

Äquivalent zum Gleichungssystem (2) sind nun die Gleichungen AG1 = 0, $\mathrm{AG} 2=0$ und $\mathrm{AG} 3=0$ :

$>\mathrm{AG} 1:=$ numer $\left(\operatorname{simplify}\left(\left((\mathrm{A} 2[1]-\mathrm{A} 1[1])^{\wedge} 2+(\mathrm{A} 2[2]-\mathrm{A} 1[2])^{\wedge} 2\right)-\mathrm{la} 2 \_2\right)\right)$ :

$>a 1:=\operatorname{coeff}\left(\mathrm{AG} 1, \mathrm{u}^{\wedge} 2\right)$ :

$>\mathrm{AG} 2:=$ numer $\left(\operatorname{simplify}\left(((\mathrm{B} 2[1]-\mathrm{B} 1[1]) \wedge 2+(\mathrm{B} 2[2]-\mathrm{B} 1[2]) \wedge 2)-1 \mathrm{~b} 2 \_2\right)\right)$ :

$>$ a2:= coeff $\left(\mathrm{AG} 2, \mathrm{u}^{\wedge} 2\right)$ :

$>\mathrm{AG} 3:=$ numer $\left(\operatorname{simplify}\left(((\mathrm{C} 2[1]-\mathrm{C} 1[1]) \wedge 2+(\mathrm{C} 2[2]-\mathrm{C} 1[2]) \wedge 2)-1 \mathrm{c} 2{ }^{\wedge} 2\right)\right)$ :

$>$ a3: $=\operatorname{coeff}\left(\mathrm{AG} 3, \mathrm{u}^{\wedge} 2\right)$ :

Es folgt die Elimination von $\mathbf{u}_{0}^{2}=u^{2}+v^{2}$ durch Differenzenbildung. Wir berücksichtigen dabei nur die Koeffizienten $a_{i}$ von $u^{2}$. Es verbleiben zwei lineare Gleichungen eq[1] und eq[2], die wir nach $u$ und $v$ auflösen:

$>$ eq $[1]:=\operatorname{eval}($ numer $(\operatorname{combine}(\mathrm{a} 1 * \mathrm{AG} 2-\mathrm{a} 2 * \mathrm{AG} 1)))$ :

$>$ eq $[2]:=\operatorname{eval}($ numer $(\operatorname{combine}(\mathrm{a} 1 * \mathrm{AG} 3-\mathrm{a} 3 * \mathrm{AG} 1)))$ :

$>\mathrm{G} 1:=\operatorname{collect}(\operatorname{expand}(\operatorname{eq}[1]),[\mathrm{u}, \mathrm{v}])$ :

$>\mathrm{G} 2:=\operatorname{collect}(\operatorname{expand}(\operatorname{eq}[2]),[\mathrm{u}, \mathrm{v}])$ :

- - Aufstellung des linearen Gleichungssystems für u und v:

$>P:=\operatorname{coeff}(\mathrm{G} 1, \mathrm{u}): \mathrm{Q}:=\operatorname{coeff}(\mathrm{G} 1, \mathrm{v}):$

$>\mathrm{S}:=\operatorname{coeff}(\mathrm{G} 2, \mathrm{u}): \mathrm{T}:=\operatorname{coeff}(\mathrm{G} 2, \mathrm{v}):$

$>\mathrm{R}:=-\operatorname{subs}(\mathrm{u}=0, \mathrm{v}=0, \mathrm{G} 1): \mathrm{U}:=-\operatorname{subs}(\mathrm{u}=0, \mathrm{v}=0, \mathrm{G} 2)$ :

$>$ mat: $=$ matrix $(2,2,[\mathrm{P}, \mathrm{Q}, \mathrm{S}, \mathrm{T}])$ :

$>$ vec: $=[\mathrm{R}, \mathrm{U}]:$

- - - Lösung des linearen Gleichungssystems:

$>$ loesung:=linsolve (mat, vec):

$>$ uu:=loesung [1]:

$>\mathrm{Vv}:=$ loesung $[2]$ :

- - Diese Lösung wird in die linke Seite AG1 der ersten Gleichung eingesetzt:

$>$ tt: $=$ numer $(\operatorname{simplify}(\operatorname{subs}(\mathrm{u}=\mathrm{uu}, \mathrm{v}=\mathrm{vv}, \mathrm{AG} 1)))$ :

Hinsichtlich der Lösbarkeit dieses Gleichungssystems ist der Kommentar in [2] auf Seite $56 \mathrm{zu}$ beachten. 


\subsection{Die Hauptschleife im Maple-Programm}

Wir bezeichnen die einzelnen Werte der Antriebswinkel für die Kurbeln $A_{0} A_{1}$, $B_{0} B_{1}$ bzw. $C_{0} C_{1}$ der Reihe nach mit ff1[i], ff1 $[i]$ bzw. ff3 $[i], i=1, \ldots$, anz, $($ anz: $=200:)$ und beginnen mit der Hauptschleife des Programms:

$>$ for $i$ from 0 to anz do

$>f f 1[i]:=s \operatorname{tart}+i * w:$

$>$ ff $2[i]:=$ omegab*ff $1[i]+$ phasb:

$>f f 3[i]:=f f 1[i]+$ phasc:

$>$ tt_werte $[i]:=[f \operatorname{solve}(\operatorname{evalf}(\operatorname{subs}(f 1=\tan (f f 1[i] / 2), f 2=\tan (f f 2[i] / 2)$, $f 3=\tan (f f 3[i] / 2), t t)))]$;

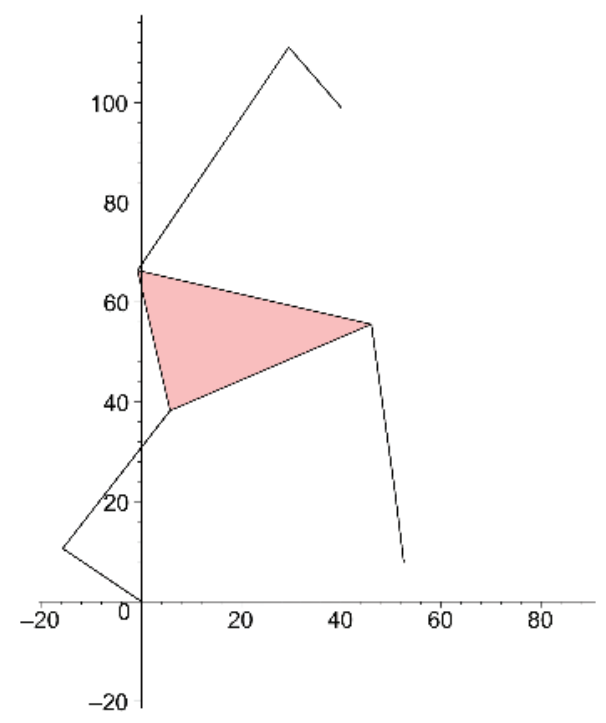

Abbildung 5. Eine mögliche Position der Gangebene $\Sigma_{4}$ des gegensinnigen F-Mechanismus zum Antriebswinkel $t=146^{\circ}$, die Drehwinkel $\varphi_{70}$ der Gangebene sind der Reihe nach $-75.688^{\circ},-55.562^{\circ},-18.443^{\circ}$ sowie $-0.982^{\circ}$ (siehe Abb.6 und Abb.7)

Bei $i=0$ wählen wir eine der Nullstellen willkürlich aus (siehe Abb. 5).

$>$ t_neu [0] :=tt_werte [0] [idx0] :

Andernfalls fragen wir die Anzahl der Nullstellen ab mittels

$>\operatorname{nbr}[i]:=\operatorname{nops}($ tt_werte $[i])$;

und suchen für festes $i$ diejenige Lösung tt_werte $[i][j], 1 \leq j \leq \operatorname{nbr}[i]$, welche dem aus der vorangegangenen Position berechneten Näherungswert naeh $[i]:=$ $t_{\text {neu }}[i-1]+d t_{\text {neu }}[i-1]$ am nächsten kommt. Dies geschieht wie folgt: 


$$
\text { "02-can" — 2015/5/21 — 23:29 — page } 31 \text { — \#11 }
$$

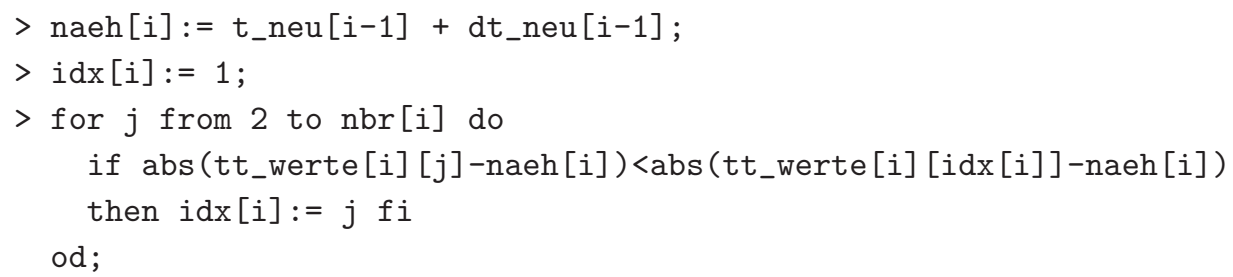

Danach setzen wir

$>$ t_neu [i] :=tt_werte $[i][i d x[i]]:$

Bei der hier vorgeschlagenen Auswahl der 'richtigen' Nullstelle sind vier Umstände zu beachten:

- tt_werte $[i][j]$ sowie $t_{\text {neu }}[i]$ sind Tangenswerte von halben Drehwinkeln $\varphi_{70}$, und diese werden bei $\varphi_{70}=180^{\circ}$ unendlich groß und wechseln bei einer geringfügigen Überschreitung ihr Vorzeichen. Deshalb müssen bei tt_werte $[i][j]$ $>1$ oder tt_werte $[i][j]<-1$ die jeweiligen Kehrwerte, also die cot-Werte bei den Größenvergleichen zur Auswahl der nächstgelegenen Lösung herangezogen werden. Die in diesem Sinn verbesserte Version des Maple-Codes lautet nun

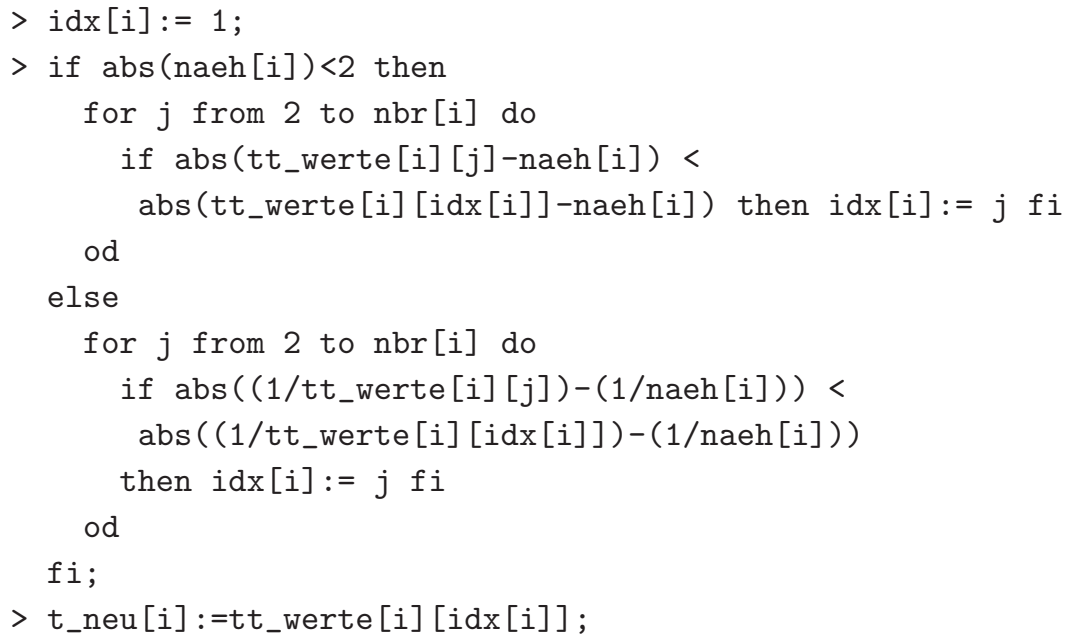

- Ist man exakt in einer singulären Lage[2], so scheitert die nachstehend gezeigte Gesch -windigkeitsanalyse wegen der verschwindenden Koeffizientendeterminante des zugehörigen linearen Gleichungssystems [2]. 
- Ist der verfolgte Zwanglauf nicht umlauffähig, so wachsen in der Nähe des Kurbelstillstandes — wenn also die Position von $\Sigma_{7}$ singulär, aber nicht zweifach singulär wird [2] — die Geschwindigkeiten über alle Grenzen. Daher wird die über die Geschwindigkeitsanalyse berechnete Näherungslösung keinen guten Vergleich ermöglichen.

- Wenn die Anzahl der Nullstellen zwischen zwei Schritten wechselt — was natürlich bedeutet, dass einer der Zwangläufe nicht umlauffähig ist —, dann wird das Programm zu einem anderen Zwanglauf wechseln, was sich in einem "Sprung" bei der Animation äußert. Bei der Getriebeanalyse mit der Getriebesoftware SAM bleibt der Mechanismus an derartigen Stellen überhaupt stehen - wie übrigens gar nicht so selten ohne erkennbaren Grund auch bei anderen Stellen, z.B., dann, wenn eine Kurbel und mit dem anschließenden Arm in einer Strecklage ist.

Als letzte Aktion innerhalb der Schleife berechnen wir einen Näherungswert für den Drehwinkel der nächsten Position. Dazu führen wir eine Geschwindigkeitsanalyse gemäß [2] durch:

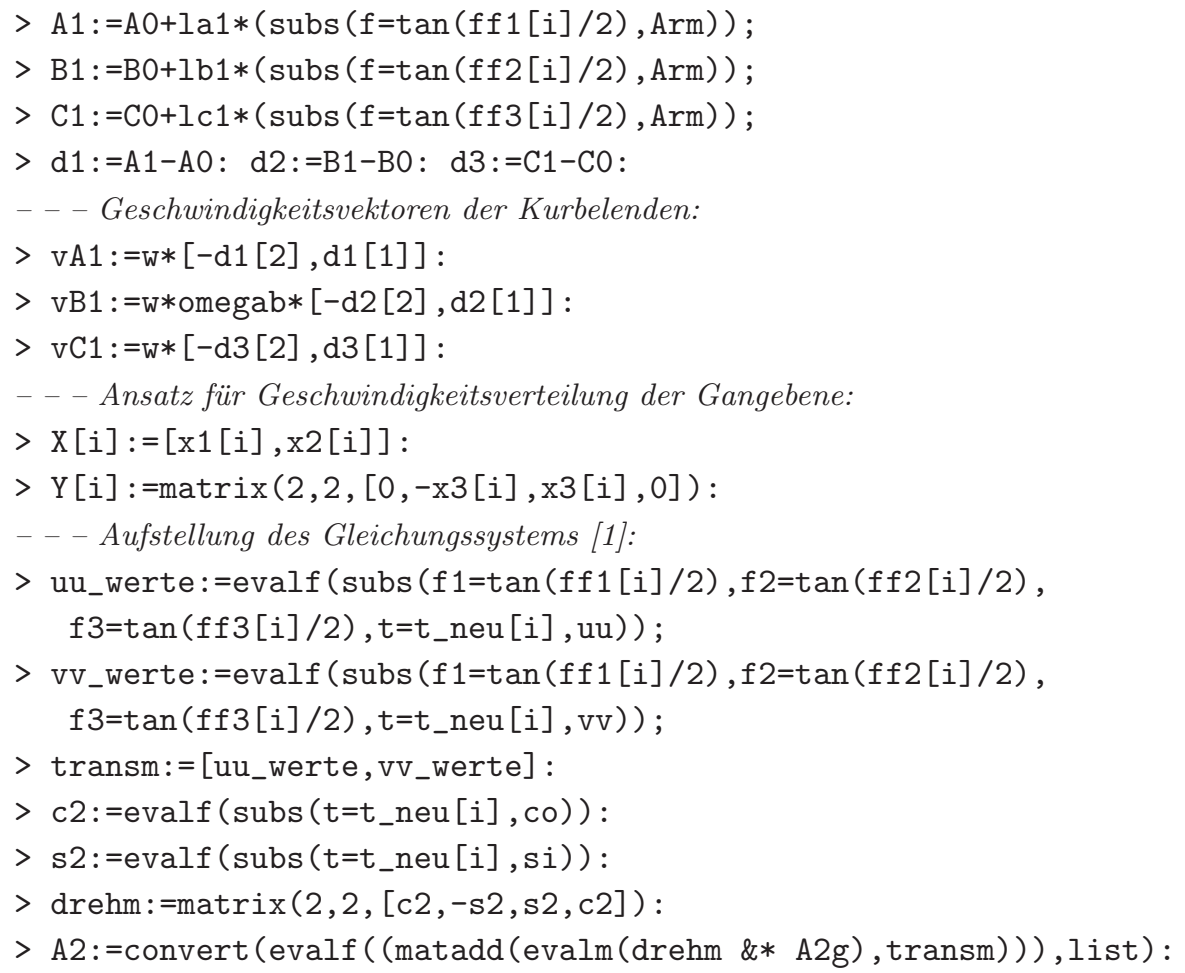



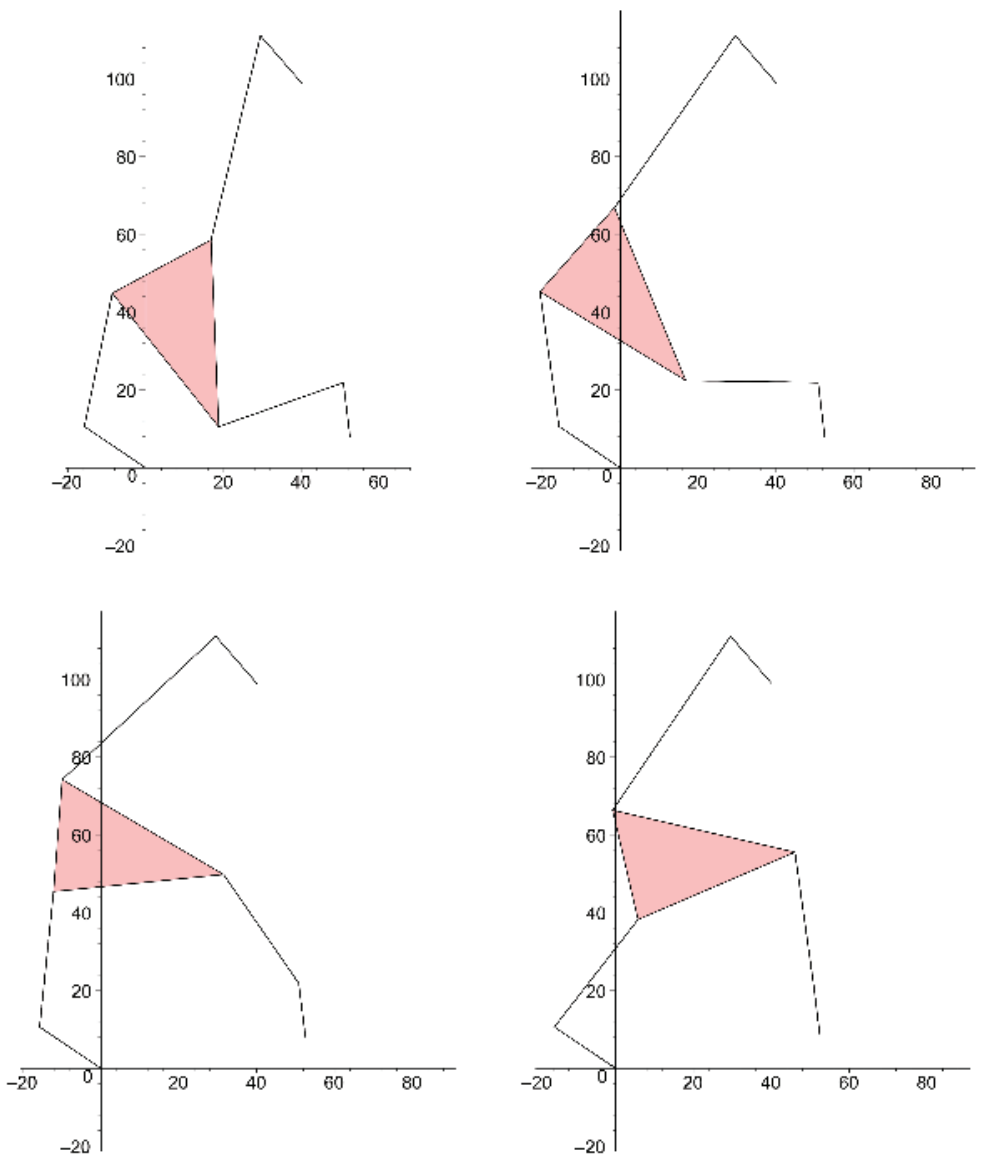

Abbildung 6. Vier verschiedene Positionen der Gangebene der Abb. 5

\footnotetext{
$>$ B2:=convert $($ evalf $((\operatorname{matadd}($ evalm $($ drehm \&* B2g $)$, transm $)))$, list $)$ :

$>\mathrm{C} 2:=\operatorname{convert}($ evalf $((\operatorname{matadd}($ evalm $(\operatorname{drehm} \& * \mathrm{C} 2 \mathrm{~g})$, transm $)))$, list $)$ :

$>\mathrm{VA} 2:=\operatorname{simplify}(\operatorname{matadd}(\operatorname{evalm}(\mathrm{Y}[\mathrm{i}] \& * \mathrm{~A} 2), \mathrm{X}[\mathrm{i}]))$ :

$>\mathrm{vB} 2:=\operatorname{simplify}(\operatorname{matadd}(\operatorname{evalm}(\mathrm{Y}[\mathrm{i}]$ \&* B2), $\mathrm{X}[\mathrm{i}]))$ :

$>\mathrm{vC} 2:=\operatorname{simplify}(\operatorname{matadd}(\operatorname{evalm}(\mathrm{Y}[\mathrm{i}] \& * \mathrm{C} 2), \mathrm{X}[\mathrm{i}]))$ :

$>\mathrm{V} 11:=\operatorname{matadd}(\mathrm{VA} 2,-\mathrm{vA} 1): \mathrm{V} 12:=\operatorname{matadd}(\mathrm{A} 2,-\mathrm{A} 1):$

$>\mathrm{V} 21:=\operatorname{matadd}(\mathrm{vB} 2,-\mathrm{vB} 1): \mathrm{V} 22:=\operatorname{matadd}(\mathrm{B} 2,-\mathrm{B} 1)$ :

$>\mathrm{V} 31:=\operatorname{matadd}(\mathrm{VC} 2,-\mathrm{vC} 1): \mathrm{V} 32:=\operatorname{matadd}(\mathrm{C} 2,-\mathrm{C} 1)$ :

$>$ GL1:=simplify (innerprod(V11,V12));
} 
$>$ GL2:=simplify (innerprod(V21,V22));

$>$ GL3:=simplify (innerprod(V31,V32));

- - Koeffizientenmatrix und Absolutspalte des Gleichungssystems:

$>$ P1: $=\operatorname{coeff}(G L 1, x 1[i]):$ P2: $=\operatorname{coeff}(G L 2, x 1[i]):$ P3:=coeff $(G L 3, x 1[i]):$

$>\mathrm{Q} 1:=\operatorname{coeff}(\mathrm{GL} 1, \mathrm{x} 2[\mathrm{i}]): \mathrm{Q} 2:=\operatorname{coeff}(\mathrm{GL} 2, \mathrm{x} 2[\mathrm{i}]): \mathrm{Q} 3:=\operatorname{coeff}(\mathrm{GL} 3, \mathrm{x} 2[\mathrm{i}]):$

$>\mathrm{R} 1:=\operatorname{coeff}(\mathrm{GL} 1, \mathrm{x} 3[\mathrm{i}]): \mathrm{R} 2:=\operatorname{coeff}(\mathrm{GL} 2, \mathrm{x} 3[\mathrm{i}]): \mathrm{R} 3:=\operatorname{coeff}(\mathrm{GL} 3, \mathrm{x} 3[\mathrm{i}])$ :

$>\mathrm{T} 1:=-\operatorname{subs}(\mathrm{x} 1[\mathrm{i}]=0, \mathrm{x} 2[\mathrm{i}]=0, \mathrm{x} 3[\mathrm{i}]=0, \mathrm{GL} 1)$ :

$>\mathrm{T} 2:=-\operatorname{subs}(\mathrm{x} 1[\mathrm{i}]=0, \mathrm{x} 2[\mathrm{i}]=0, \mathrm{x} 3[\mathrm{i}]=0, \mathrm{GL} 2)$ :

$>\mathrm{T} 3:=-\operatorname{subs}(\mathrm{x} 1[\mathrm{i}]=0, \mathrm{x} 2[\mathrm{i}]=0, \mathrm{x} 3[\mathrm{i}]=0, \mathrm{GL} 3)$ :

$>$ mat:=matrix $(3,3,[\mathrm{P} 1, \mathrm{Q} 1, \mathrm{R} 1, \mathrm{P} 2, \mathrm{Q} 2, \mathrm{R} 2, \mathrm{P} 3, \mathrm{Q} 3, \mathrm{R} 3])$ :

$>$ vec: $=[\mathrm{T} 1, \mathrm{~T} 2, \mathrm{~T} 3]:$

- - - Bestimmung der Lösung:

$>$ losung:=linsolve(mat, vec):

$>\mathrm{x} 1[\mathrm{i}]:=$ losung[1]: $\mathrm{x} 2[\mathrm{i}]:=$ losung[2]:

$>\mathrm{x} 3[\mathrm{i}]:=$ losung $[3]$ :

$>d t \_n e u[i]:=\operatorname{eval}\left(\left(1+t \_n e u[i] * t \_n e u[i]\right) * x 3[i] / 2\right)$ :

$>$ od:

Hinter der vorletzten Programmzeile steht die folgende Überlegung:

Wir suchen eine Näherungswert für $t_{\text {neu }}[i+1]$ in der Form $t_{\text {neu }}[i]+d t_{\text {neu }}[i]$ nach dem Muster

$$
f(x+h) \sim f(x)+h f^{\prime}(x)
$$

mit $x$ als Antriebswinkel $t$ und mit $h$ als Schrittweite $w$, denn $w$ war die 'Winkelgeschwindigkeit' der Antriebskurbeln im obigen Programmcode bei der Festsetzung der Geschwindigkeitsvektoren vA1, . . der Kurbelenden $A_{1}, \ldots$

Die Funktion $f$ ist $\tan \frac{\varphi}{2}$ mit $\varphi=\varphi_{70}(t)$, und dabei ist $h \dot{\varphi}_{70}$ gleich der Lösung $x_{3}$ in dem linearen Gleichungssystem zur Geschwindigkeitsanalyse. Daher ist

$$
d t_{\text {neu }}[i]=h \frac{d f}{d t}=h \frac{d}{d t}\left(\tan \frac{\varphi}{2}\right)=\left(1+\tan ^{2} \frac{\varphi}{2}\right) \frac{h \dot{\varphi}}{2}=\left(1+\left(t_{\text {neu }}[i]\right)^{2}\right) \frac{x_{3}[i]}{2} .
$$

\subsection{Verschiedene Ausgabemöglichkeiten}

Welche Graphikausgabe auch immer gewünscht wird, stets beginnt man mit der Berechnung der einzelnen Positionen, und zwar derjenigen zu den vorhin gespeicherten Drehwinkeln $t_{\text {neu }}[i]$ :

- - Berechnung der Positionen:

$>$ for i from 0 to anz do 
$>\mathrm{AA} 1[\mathrm{i}]:=\mathrm{A} 0+\operatorname{la} 1 *(\operatorname{subs}(\mathrm{f}=\tan (\mathrm{ff} 1[\mathrm{i}] / 2), \mathrm{Arm}))$;

$>\mathrm{BB} 1[i]:=\mathrm{B} 0+\operatorname{lb} 1 *(\operatorname{subs}(\mathrm{f}=\tan (\mathrm{ff} 2[\mathrm{i}] / 2), \operatorname{Arm}))$;

$>\operatorname{CC} 1[i]:=\mathrm{C} 0+1 \mathrm{c} 1 *(\operatorname{subs}(\mathrm{f}=\tan (\mathrm{ff} 3[\mathrm{i}] / 2), \mathrm{Arm}))$;

$>$ uu_werte: $=\operatorname{evalf}(\operatorname{subs}(f 1=\tan (f f 1[i] / 2), f 2=\tan (f f 2[i] / 2)$, $\left.\left.f 3=\tan (f f 3[i] / 2), t=t \_n e u[i], u u\right)\right)$;

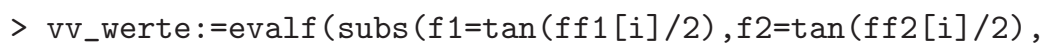
$\left.\left.\mathrm{f} 3=\tan (\mathrm{ff} 3[\mathrm{i}] / 2), \mathrm{t}=\mathrm{t} \_\mathrm{neu}[\mathrm{i}], \mathrm{vv}\right)\right)$;

- - Sollte die Koeffizientenmatrix des zugehörigen Gleichungssystems 0 sein[1], so kommt es hier zu einer Division durch 0:

$>\operatorname{trans} 2:=\left[u u_{-}\right.$werte,vv_werte];

$>c 2:=\operatorname{evalf}\left(\operatorname{subs}\left(t=t \_n e u[i], c o\right)\right):$

$>\mathrm{s} 2:=\operatorname{evalf}\left(\operatorname{subs}\left(\mathrm{t}=\mathrm{t} \_\mathrm{neu}[\mathrm{i}], \mathrm{si}\right)\right)$ :

$>$ dreh2:=matrix $(2,2,[\mathrm{c} 2,-\mathrm{s} 2, \mathrm{~s} 2, \mathrm{c} 2])$ :

$>\mathrm{AA} 2[\mathrm{i}]:=\operatorname{convert}(\operatorname{evalf}((\operatorname{matadd}(\operatorname{evalm}(\operatorname{dreh} 2 \& * \mathrm{~A} 2 \mathrm{~g}), \operatorname{trans} 2)))$, list $)$ :

$>\mathrm{BB} 2[\mathrm{i}]:=\operatorname{convert}(\operatorname{evalf}((\operatorname{matadd}(\operatorname{evalm}(\operatorname{dreh} 2 \& * \mathrm{~B} 2 \mathrm{~g}), \operatorname{trans} 2)))$, list $)$ :

$>\mathrm{CC} 2[\mathrm{i}]:=\operatorname{convert}(\operatorname{evalf}((\operatorname{matadd}(\operatorname{evalm}(\operatorname{dreh} 2 \& * \mathrm{C} 2 \mathrm{~g}), \operatorname{trans} 2)))$, list $)$ :

- - Die Schleife ist noch nicht abgeschlossen!

Soll mit Maple eine Animation des Zwanglaufes erzeugt werden, muss man nach der Berechnung der Positionen innerhalb der Schleife wie folgt vorgehen:

- - Es werden die Elemente der Animation definiert:

$>$ Dreieck[i] :=polygonplot ([AA2 [i], BB2 [i], CC2 [i]], color=red):

$>$ Gelenk_a01[i]:=polygonplot ([A0,AA1[i]],thickness=2) :

$>$ Gelenk_a12[i]:=polygonplot ([AA1[i], AA2[i]], thickness=2) :

$>$ Gelenk_b01[i]:=polygonplot ([BO,BB1 [i] ], thickness=2):

$>$ Gelenk_b12[i]:=polygonplot ([BB1[i], BB2[i]], thickness=2):

$>$ Gelenk_c01[i]:=polygonplot ([C0, CC1 [i] ], thickness=2):

$>$ Gelenk_c12[i]:=polygonplot ([CC1 [i], CC2 [i] ], thickness=2) :

$>$ od:

- - Ende der Schleife:

> Konfiguration:=seq (display (Dreieck[i], Gelenk_a01 [i],Gelenk_b01[i], Gelenk_c01[i], Gelenk_a12[i], Gelenk_b12[i], Gelenk_c12[i]), $i=1$. . anz):

- - Befehl zur Ausgabe der Animation:

> display (Konfiguration, scaling=constrained, insequence=true);

Die hier erfolgte Trennung der Streckenzüge $A_{0} A_{1} A_{2}$ usw. in jeweils zwei Zeilen hat den alleinigen Grund darin, dass die verfügbare Version von Maple hier zusätzliche Geraden einfügt, die dann den Betrachter eher verwirren. Der Befehl 'scaling=constrained' garantiert ein maßtabsgetreues Bild. 
Eine Darstellung der Punktbahnen (Abb. 3) gelingt bei der verwendeten Maple-Version (Maple 13) durch folgenden Trick: Wir speichern bei der Berechnung der Ecken $A_{2}[i], \ldots$ des Gangdreiecks immer auch den jeweiligen Vorgänger mit ab und lassen anschließend die Verbindungssehnen zeichnen.

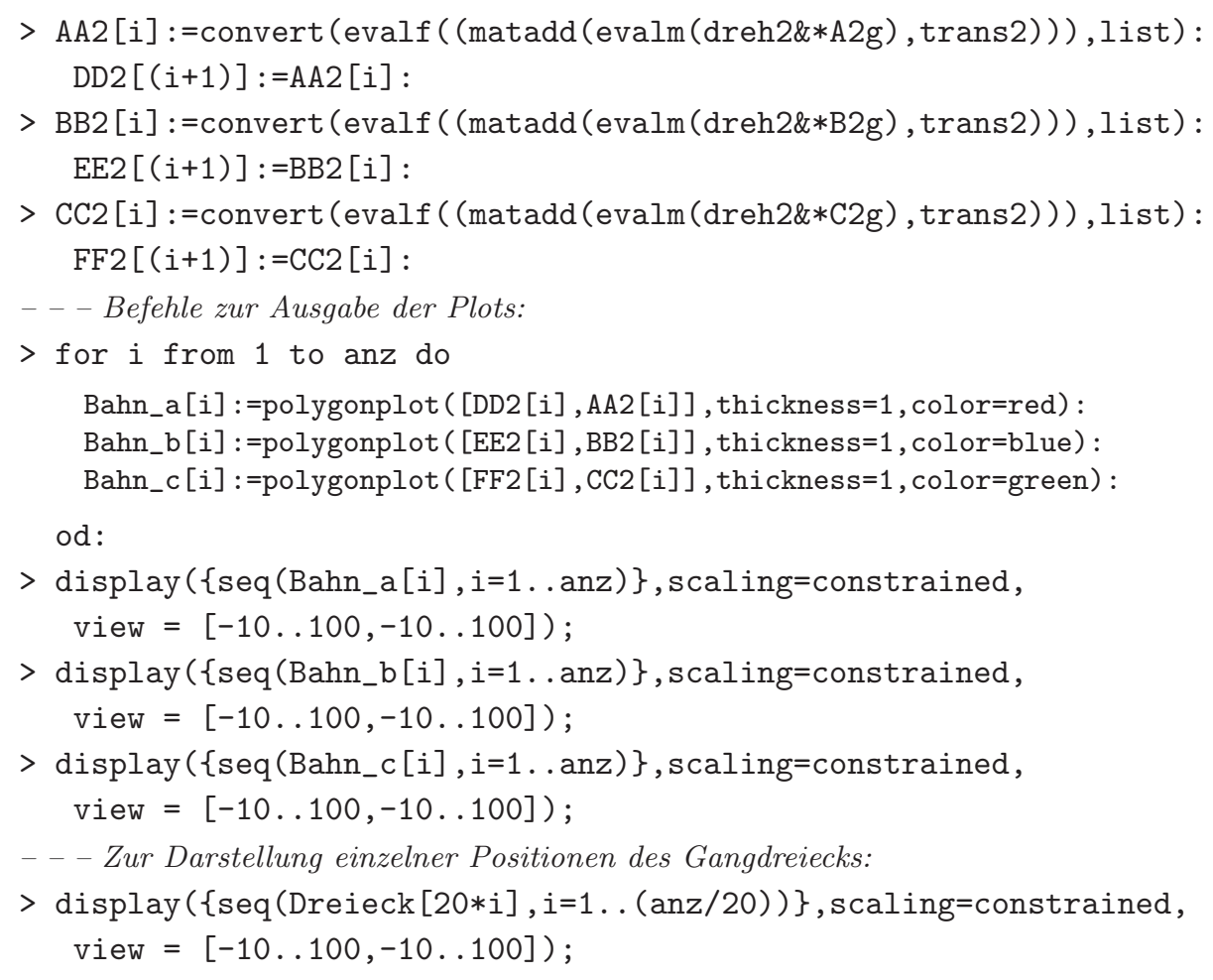

Auch hier wurden die Bahnen getrennt voneinander ausgegeben, um zu vermeiden, dass Maple zusätzliche Streckenbilder einfügt.

Will man eine Übersicht darüber haben, zu welchen Antriebswinkeln (hier mit ff1[i] bezeichnet) wieviele Positionen der Gangebene $\Sigma_{7}$, also wieviele Drehwinkel $\varphi_{70}$ möglich sind, kann man die zu diskreten $f f_{1}$-Werten gehörigen $\varphi_{70}$-Wert (hier tt_werte $[i][j]$ ) — jeweils im Gradmaß - in einem Diagramm zeichnen lassen (siehe Abb.7). Zuvor muss man allerdings ff1 auf das Intervall [0,2 $\pi]$ normieren:

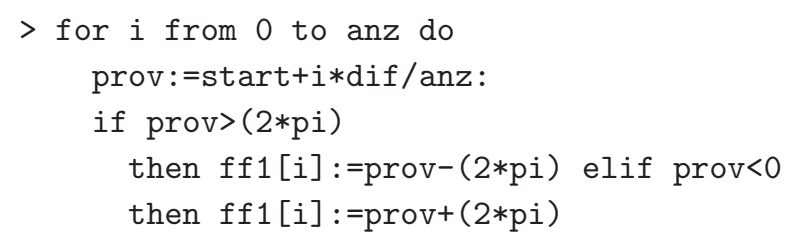




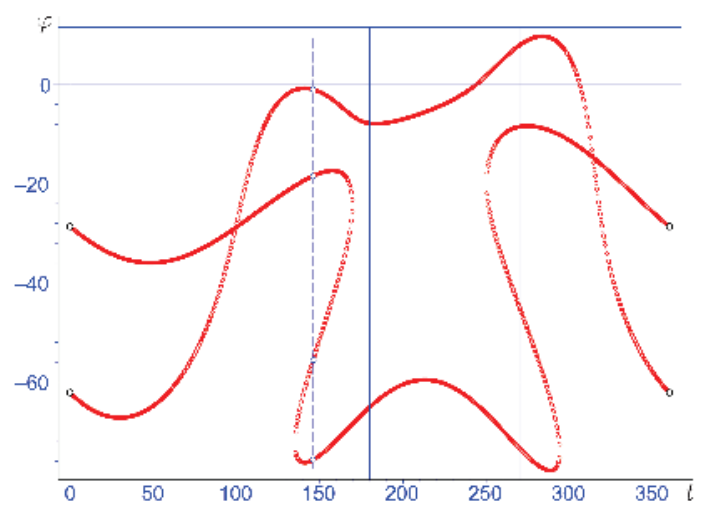

Abbildung 7. Drehwinkel $\varphi_{70}$ als Funktion des Antriebswinkels t zu den Daten des gegensinnigen F-Mechanismus aus dem Beispiel 2 (siehe auch Abb. 6). Dort werden die vier Positionen des Gangdreiecks zum Antriebswinkel $t=146^{\circ}$ gezeigt.

else $f f 1[i]:=p r o v$

end if;

od:

Es war bei Maple 13 nicht möglich, das Diagramm direkt zeichnen zu lassen, wenn zu verschiedenen $t$-Werten unterschiedliche Lösungsanzahlen auftreten. Deshalb wurde die Maximalanzahl loesg der jeweils auftretenden Lösungen ermittelt, und dort, wo die tatsächlich Lösungsanzahl geringer war, wurde das Array der $\varphi_{70}$-Werte mit Nullen aufgefüllt:

$>\operatorname{grd}:=180 / \mathrm{pi}$ :

$>$ for $i$ from 1 to anz do

$\mathrm{AA} 1[i]:=\mathrm{A} 0+\operatorname{la} 1 *(\operatorname{subs}(\mathrm{f}=\tan (\mathrm{ff} 1[\mathrm{i}] / 2), \mathrm{Arm})):$

$\mathrm{BB} 1[\mathrm{i}]:=\mathrm{B} 0+\mathrm{lb} 1 *(\operatorname{subs}(\mathrm{f}=\tan (\mathrm{ff} 2[\mathrm{i}] / 2), \mathrm{Arm})):$

$\mathrm{CC} 1[i]:=\mathrm{C} 0+1 \mathrm{c} 1 *(\operatorname{subs}(\mathrm{f}=\tan (\mathrm{ff} 3[i] / 2), \operatorname{Arm}))$ :

for $j$ from 1 to $n b r[i]$ do

$\mathrm{XX}[i, j]:=\left[\mathrm{ff} 1[i] * \operatorname{grd}, \arctan \left(t t_{-}\right.\right.$werte $\left.\left.[i][j]\right) * 2 * \operatorname{grd}\right]$;

od;

if $\mathrm{nbr}[\mathrm{i}]<$ loesg then

for $j$ from $(n b r[i]+1)$ to loesg do

$X X[i, j]:=[0,0]$;

od

end if; 
od:

- - Wir wandeln das zweidimensionale Array der XX in ein eindimensionales:

$>$ for $i$ from 1 to anz do

for $j$ from 1 to loesg do

$\mathrm{YY}[(i-1) *$ loesg $+j]:=\mathrm{XX}[i, j]$;

od

od;

$>\operatorname{pointplot}(\{\operatorname{seq}(Y Y[i], i=1 \ldots(\operatorname{anz} *$ loesg $))\}$, color=red, axes=boxed, symbol=CIRCLE, symbolsize=8);

Die Diagrammpunkte mit vertikalen Tangenten gehören zu Umkehrlagen der Antriebskurbeln, sofern nicht die Tangente die Diagrammkurve hier durchsetzt. Die Doppelpunkte (Selbstschnitte) der Diagrammkurve bestimmen Verzweigungslagen des F-Mechanismus, sofern nicht an dieser Stelle die Koeffizientendeterminante des linearen Gleichungssystems (2) für $\mathbf{u}_{0}=\left(u_{x}, u_{y}\right)$ verschwindet.

Um so wie in Abb. 8 den Verlauf aller möglichen Positionen der Ecken des Gangdreiecks darstellen zu lassen, gibt es folgende Möglichkeit:

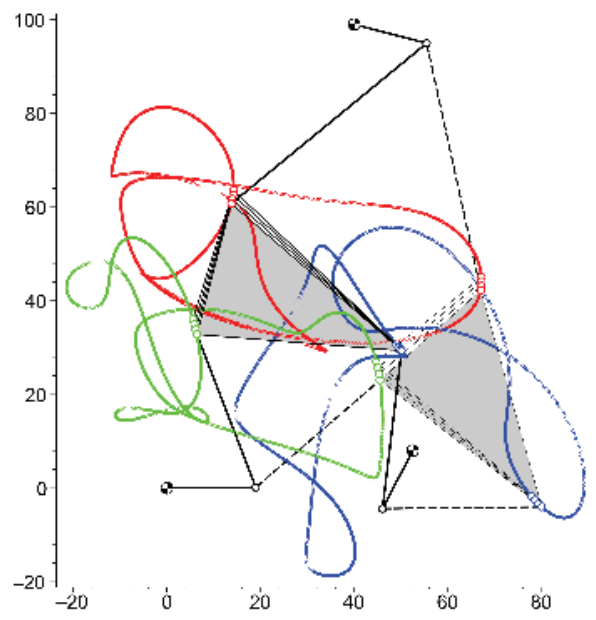

Abbildung 8. Bahnen der Ecken des Gangdreiecks bei den Positionen des gegensinnigen F-Mechanismus aus Beispiel 2 (siehe auch Abb.7). Die zum Antriebswinkel $0.5^{\circ}$ gehörigen beiden Ausgangslagen sind besonders hervorgehoben. Die strichlierte Lage gehört zu dem umlauffähigen Zwanglauf, der auch in Abb.3 dargestellt ist.

$>$ aa: $=$ pointplot $(\{\operatorname{seq}(\mathrm{AA}[i], i=1 \ldots($ anz*loesg $))\}$, color=blue, axes=boxed, symbol=CIRCLE, symbolsize $=6$, scaling=constrained): 


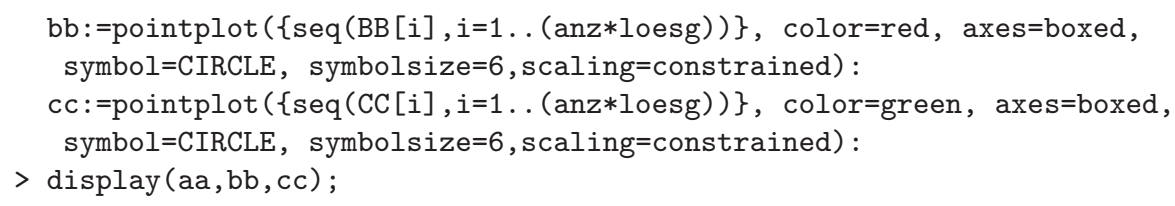

Die Abbildung 8 zeigt ein Beispiel dazu.

$\mathrm{Zu}$ einer Auswahl der jeweiligen Zusammenhangskomponenten und damit zum Zeichnen der eigentlichen Bahnkurven (so wie in Abb.3) kommt man nur, wenn man bei der numerischen Berechung der nächsten Position die Geschwindigkeitsanalyse aus der vorhergehenden Lage heranzieht.

\section{Literatur}

[1] E. Can and H. Stachel, A planar parallel 3-RRR robot with synchronously driven cranks, Mech. and Mach. Theory 79 (2014), 29-45.

[2] E. Can, Analyse and Synthese eines schnelllaufenden ebenen Mechanismus mit modifizierbaren Zwangläufen, PhD Thesis, Vienna University of Technology, 2012.

[3] S. Ahamed, Simulation of a 3-DOF 3-RRR Planar Parallel Robot, www . parallemic .org/Java/3RRR.html.

[4] W. Gander and J. Hrebicek, Solving Problems in Scientific Computing Using Maple and Matlab, Heidelberg Springer, Berlin, 2004.

[5] S. Staicu, Kinematics of the 3-RRR planar parallel robot, U.P.B. Sci. Bull. 70, no. 2, Ser. D (2008), 3-14.

[6] W. Wunderlich, Ebene Kinematik, BI-Hochschultaschenbücher, Bd. 447, Bibliographisches Institut, Mannheim (1970).

ENGIN CAN

KAYNARCA SCHOOL OF APPLIED SCIENCES

SAKARYA UNIVERSITY

TR54650 SAKARYA

TURKEY

E-mail: ecan@sakarya.edu.tr

(Received June, 2014) 